\title{
Spherical cuts for Integer Programming problems
}

\author{
LEO LIBERTI \\ LIX, École Polytechnique, F-91128 Palaiseau, France \\ (liberti@lix.polytechnique.fr)
}

June 25, 2007

\begin{abstract}
We introduce a new family of valid inequalities for general linear integer programming problems, based on the distance of the relaxed solution to the closest integral point. We show that these are valid cuts, establish some relations with Balas' intersection cuts, and show that a straightforward cutting plane algorithm derived from either spherical or intersection cuts will in general only converge if a suitable Gomory-type strengthening is put in place.
\end{abstract}

Keywords: valid cut, cutting plane algorithm, intersection cuts, integer programming.

\section{Introduction}

In this paper we propose a new family of valid cuts for Integer Programming (IP) linear problems in the following general form:

$$
\left.\begin{array}{cl}
\min _{x} & c x \\
\text { s.t. } & A x \leq b \\
& x \geq 0 \\
& x \in \mathbb{Z}^{n},
\end{array}\right\}
$$

where $x \in \mathbb{Z}^{n}$ are the decision variables, $c \in \mathbb{R}^{n}$ is a row cost vector, $A$ is an $m \times n$ matrix and $b \in \mathbb{R}^{m}$.

Let $P=\left\{x \in \mathbb{Z}_{+}^{n} \mid A x \leq b\right\}$ be the feasible region of problem (1), and let $R=\left\{x \in \mathbb{R}_{+}^{n} \mid A x \leq b\right\}$ be the continuous relaxation of the feasible region $P$. Assume $R$ has a non-empty interior and that $\operatorname{dim} \operatorname{aff}(R)=n$. Let $x^{*}$ be the (integral) solution of $(1)$, let $x^{\prime}$ be the solution of the relaxed problem $\min _{x \in R} c x$ and assume that $x^{\prime}$ is not an integral vector. The cuts we shall propose are suitable for use in a cutting plane or Branch-and-Cut algorithm.

Finding valid cuts is a fundamental task when solving IP problems. The most effective cutting techniques usually rely on problem structure. See [15], Ch. II.2 for a good technical discussion on the 
most standard techniques, and $[13,14,10]$ for recent interesting group-theoretical approaches which are applicable to large subclasses of IPs. Valid cuts for IP problems in general form (1) are not as easy to come by. Valid inequalities are generated by all relaxation hierarchies (like e.g. Sherali-Adams' [16]). The best known general-purpose valid cuts are the Gomory cuts [8]: they are simple to define, can be written in a form suitable for straightforward insertion in a simplex tableau, and are guaranteed to yield the optimal integer solution in a finite number of steps of a cutting plane algorithm; many strengthenings of the Gomory cutting planes have been proposed [11]. Lift-and-project techniques are used to generate new cuts from existing inequalities [4]. Families of valid cuts for general Binary Integer Programming (BIP) problems have been derived, for example, in [5, 12], based on geometrical properties of the definition hypercube $\{0,1\}^{n}$. In [5], inequalities defining the various faces of the unit hypercube are derived. The cuts proposed in [12] are defined by finding a suitable hyperplane separating a unit hypercube vertex $\bar{x}$ from its adjacent vertices. Balas' intersection cuts [2], based on finding intersection points between the hypersphere circumscribing the unit hypercube and the extreme rays of a cone rooted at the current relaxed optimum, are investigated in Section 3. In [6], Fenchel duality arguments are used to find the maximum distance between the solution of the continuous relaxation of (1) and the convex hull of the feasible set; this gives rise to provably deep cuts (called "Fenchel cuts"). The constraint programming community has developed techniques for generating valid cuts which are based on logical inference methods $[9,1,7]$. Although these cuts may not always be expressed as linear inequalities, they are nonetheless rules which attempt to separate the solution of the relaxation from the integral feasible region.

The main contribution of this paper is the description of a new family of cutting planes, called spherical cuts, which can be applied to linear IPs in general form. These are based on the following geometrical observation (described graphically in Fig. 1). Consider the ball centered at $x^{\prime}$ with radius $\left\|x^{\prime}-\bar{x}\right\|$, where $\bar{x}$ is the integral vector nearest to $x^{\prime}$. Intuitively, we can discard all the points in the interior of this ball as they will not be integer (thus defining an improved, albeit nonconvex, feasible region). The cuts we propose define a tighter convex relaxation of the improved feasible region.

Section 2 contains the definitions and theoretical results concerning spherical cuts. Section 3 establishes some relations between Balas' intersection cuts and spherical cuts. Section 4 shows that a straightforward cutting plane algorithm based on spherical or intersection cuts need not converge unless a Gomory-type cut modification is employed. Section 5 concludes the paper.

\section{Spherical cuts}

By a valid cut we mean an inequality which is valid for $P$ but which is not satisfied by at least a nonintegral point of $R$. Notationally, given a set of constraint indices $I$ we indicate with $A_{I}$ the matrix 
formed by the rows of $A$ indexed by $I$. Likewise, $b_{I}$ is the column vector formed by the rows of $b$ indexed by $I$. We make use of the fact that $x^{*}$ is a vertex of the polyhedron $R$ if and only if $x^{*}$ may not be written as a strict convex combination of points of $R$.

Let $x^{\prime}$ be the solution of the continuous relaxation of (1) corresponding to a vertex of the polyhedron $R$, assume that $x^{\prime}$ is not an integral vector, and let $\bar{x}$ the integral vector nearest to $x^{\prime}$ (this can be found by rounding each fractional component of $x^{\prime}$ to the nearest integer value). Let $\gamma=\left\|x^{\prime}-\bar{x}\right\|$. Since we assumed that $x^{\prime}$ is not integral, we have $\gamma>0$.

\subsection{Proposition}

The nonconvex inequality

$$
\left\|x-x^{\prime}\right\| \geq \gamma
$$

is a valid cut for $P$.

Proof. By definition $\gamma=\min \left\{\left\|x-x^{\prime}\right\| \mid x \in \mathbb{Z}^{n}\right\}$, so all the points in $P$ are feasible with respect to (2). However, $x^{\prime} \in R$ is not feasible with respect to (2) because $\left\|x^{\prime}-x^{\prime}\right\|=0<\gamma$, as claimed.

So (2) is a nonconvex valid cut for problem (1). Let $Q=R \cap\left\{x \mid\left\|x-x^{\prime}\right\| \geq \gamma\right\}$. A convex relaxation for $Q$ can easily be obtained by identifying $n$ points $\beta_{1}, \ldots, \beta_{n}$ where the spherical surface $S\left(x^{\prime}, \gamma\right)$ intersects $n$ edges of the polyhedron $R$ adjacent to $x^{\prime}$ (see the example in Fig. 1 and see also the discussion of the case when $x^{\prime}$ is a degenerate vertex in the next paragraph). The points $\beta_{1}, \ldots, \beta_{n}$ define a hyperplane $\pi x \leq \pi_{0}$ which is a valid (linear) inequality for $P$ and a valid cut with respect to $R$. We call such a cut a spherical cut.

More precisely, let $I^{\prime}$ be the set of indices of constraints which are active at $x^{\prime}$. Since $x^{\prime} \in \mathbb{R}^{n}$ and $x^{\prime}$ is a vertex of $R$, we have $\left|I^{\prime}\right| \geq n$. Let $I$ be a subset of $I^{\prime}$ such that $|I|=n$ and $A_{I}$ is nonsingular. Then

$$
A_{I} x^{\prime}=b_{I}
$$

This corresponds to a choice of active constraints whose gradients are linearly independent; some of these constraints may be range constraints and may not have a corresponding row in the canonical constraint matrix. For each $i \leq n$ define $I(i)=I \backslash\{i\}$. The $n$ linear systems $A_{I(i)} x=b_{I(i)}$ (each having rank $n-1$ ) for $i \leq n$ describe $n$ one-dimensional affine spaces which contain $x^{\prime}$. For each $i \leq n$, let $d_{i}$ be a unit direction vector satisfying $A_{I(i)} d_{i}=0$ such that $\beta_{i}=x^{\prime}+\gamma d_{i}$ satisfies $A_{I} \beta_{i} \leq b_{I}$. If $x^{\prime}$ is a nondegenerate vertex, all these spaces contain an edge of $R$ which is adjacent to $x^{\prime}$. For example in Fig. 1 there are two such affine spaces, i.e. those containing the segments $\left(x^{\prime}, \beta_{1}\right)$ and $\left(x^{\prime}, \beta_{2}\right)$, leading to the construction of the spherical cut $\pi x \leq \pi_{0}$. If $x^{\prime}$ is degenerate, some of these spaces may not contain edges of $R$, but the derived spherical cut is valid nonetheless (see Fig. 2). 


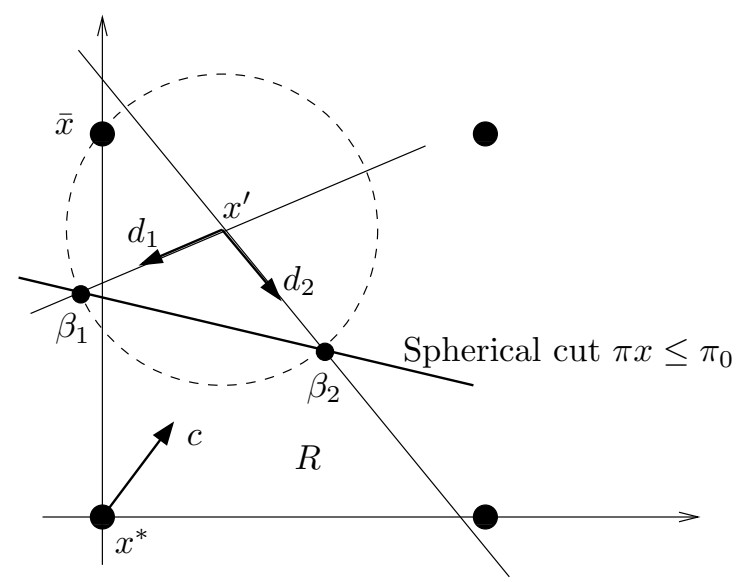

Figure 1: The spherical cut construction in 2D.

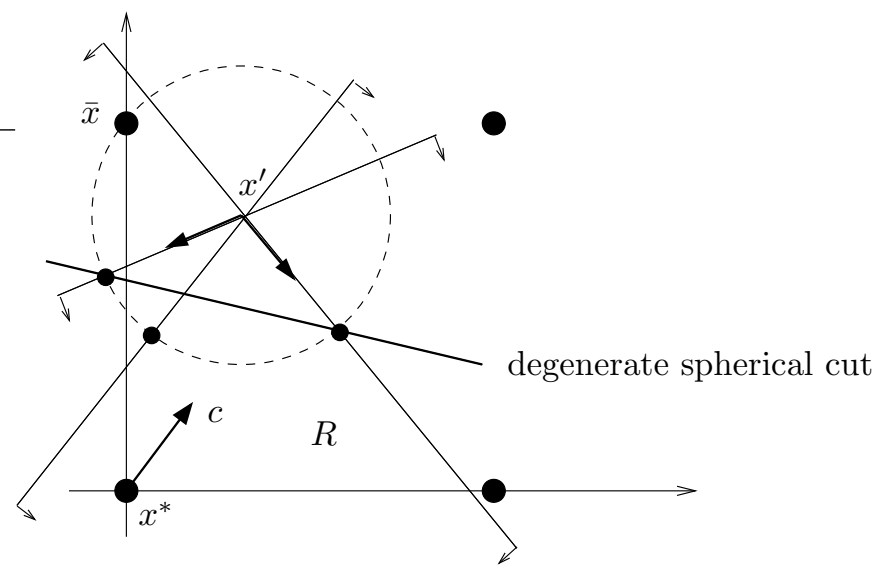

Figure 2: Spherical cut on degenerate vertex.

\subsection{Theorem}

Let $\pi x=\pi_{0}$ be the hyperplane passing through $\beta_{1}, \ldots, \beta_{n}$. Then $\pi x=\pi_{0}$ separates $x^{\prime}$ from $\operatorname{conv}(P)$.

Proof. Let $Q_{0}=\left\{x \mid \pi x \leq \pi_{0}\right\}$ and $\bar{Q}=R \cap Q_{0} . \bar{Q}$ is easily shown to be a convex relaxation of $Q$ (it is convex by definition and it contains every point of $Q$ by construction). Hence, the inequality $\pi x \leq \pi_{0}$ is valid for each point in $Q$. Suppose now, to get a contradiction, that $\pi x^{\prime}=\pi_{0}$. This means that the directions $d_{i}$ are coplanar, and hence affinely dependent. So there are affine coefficients $\lambda_{1}, \ldots, \lambda_{n}$ with $\sum_{i=1}^{n} \lambda_{i}=1$ such that $\sum_{i=1}^{n} \lambda_{i} d_{i}=0$. Pick $\varepsilon>0$ such that $\xi_{i}=x^{\prime}+\varepsilon d_{i} \in R$ for all $i \leq n$. We have:

$$
\sum_{i=1}^{n} \lambda_{i} \xi_{i}=\sum_{i=1}^{n} \lambda_{i}\left(x^{\prime}+\varepsilon d_{i}\right)=x^{\prime} \sum_{i=1}^{n} \lambda_{i}+\varepsilon \sum_{i=1}^{n} \lambda_{i} d_{i}=x^{\prime} .
$$

So $x^{\prime}$ can be expressed as a (strict) affine combination of the points $\xi_{1}, \ldots, \xi_{n}$, all of which are contained 
in the polyhedron $R$. So $x^{\prime}$ cannot not a vertex of $R$, against the assumption.

An alternative proof to Thm. 2.2 is provided by an easy corollary to Thm. 1 in [3] (attributed to F. Glover).

\section{Intersection cuts}

Intersection cuts [2] are general-purpose valid cuts for IP problem introduced by Balas in 1971. Let $x^{\prime}$ be the solution of the continuous relaxation of (1) and $\bar{x}$ be the closest integral vector to $x^{\prime}, e=\left(\frac{1}{2}, \ldots, \frac{1}{2}\right) \in$ $\mathbb{R}^{n}, y=\left\lfloor x^{\prime}\right\rfloor+e$ and $\gamma_{0}=\|y-\bar{x}\|=\frac{\sqrt{n}}{2}$. Then it is easy to show that $\|x-y\| \geq \gamma_{0}$ is a (nonconvex) valid inequality for (1). Let $Q_{0}=R \cap\left\{x \mid\|x-y\| \geq \gamma_{0}\right\}$. A convex relaxation for $Q_{0}$ can be obtained with the same techniques as those used to obtain the convex relaxation for $Q$ in Sect. 2, as illustrated by Fig. 3.

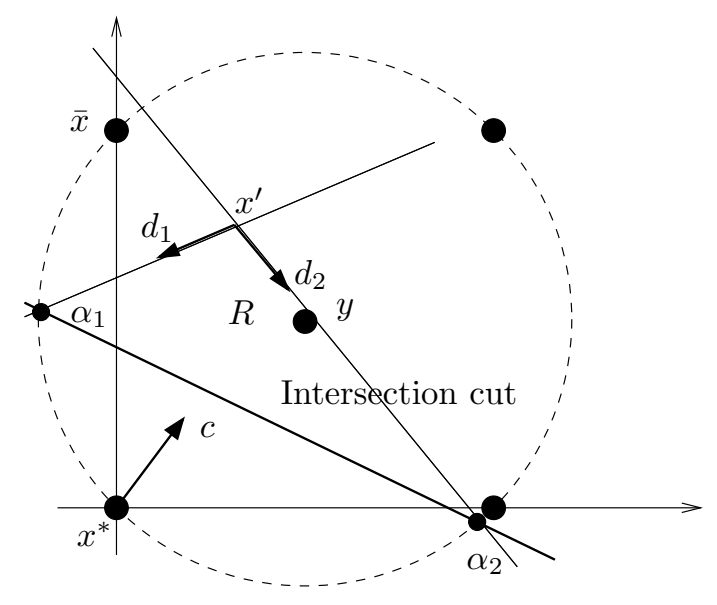

Figure 3: An intersection cut.

In particular, intersection cuts are based on the intersection of the semi-infinite lines $x^{\prime}+f_{i}$ (for $i \leq n)$ with the hypersphere circumscribing the unit hypercube containing $x^{\prime}$, where $f_{i}$ is the $R$-feasible direction defined by $A_{I(i)} f_{i}=0$. This is the same construction as for spherical cuts, the only difference being in how the sphere is defined. We denote by $\sigma$ be the sphere with equation $\|x-y\|=\|\bar{x}-y\|$ (defining sphere for intersection cuts) and $S$ be the sphere with equation $\left\|x-x^{\prime}\right\|=\left\|\bar{x}-x^{\prime}\right\|$ (defining sphere for spherical cuts). We can now use Lemma 2 in [2] to find $\alpha_{i}=x^{\prime}+f_{i}$ for all $i \leq n$ : we write $f_{i}=\lambda_{i} \bar{a}_{i}$ where $\bar{a}_{i}$ is the $i$-th column of the final simplex tableau (completed with zeroes in components corresponding to nonbasic variables). For intersection cuts, $\lambda_{i}$ is a steplength defined as follows (for all $i \leq n)$ :

$$
\forall i \leq n \quad \lambda_{i}=\frac{\left(x^{\prime}-\left\lfloor x^{\prime}\right\rfloor-e\right) \bar{a}_{i}}{\left\|\bar{a}_{i}\right\|^{2}}+\frac{\|e\|}{\left\|\bar{a}_{i}\right\|}
$$


We remark that (4) is derived from Eq. (12) in [2] by simple transformations. For spherical cuts, as was mentioned above, we write $\beta_{i}=x^{\prime}+\gamma d_{i}$ where $d_{i}=\frac{\bar{a}_{i}}{\left\|\bar{a}_{i}\right\|}$ and $\gamma=\left\|x^{\prime}-\bar{x}\right\|$, for all $i \leq n$. We can use Thm. 2 in [2] to compute the cut coefficients.

\subsection{Dominance relations}

It turns out there is no dominance relation between the class of intersection cuts and that of spherical cuts. A valid inequality $\pi x \leq \pi_{0}$ is defined to be dominant in $R$ with respect to another valid inequality $\mu x \leq \mu_{0}$ if there is a value $u>0$ such that $u \mu \leq \pi$ and $\pi_{0} \leq u \mu_{0}$. Fig. 4 shows an example where an intersection cut dominates a spherical cut. On the other hand, it is possible to construct cases where there is no dominance relation (see Fig. 5) and cases where spherical cuts dominate intersection cuts (see Fig. 6).

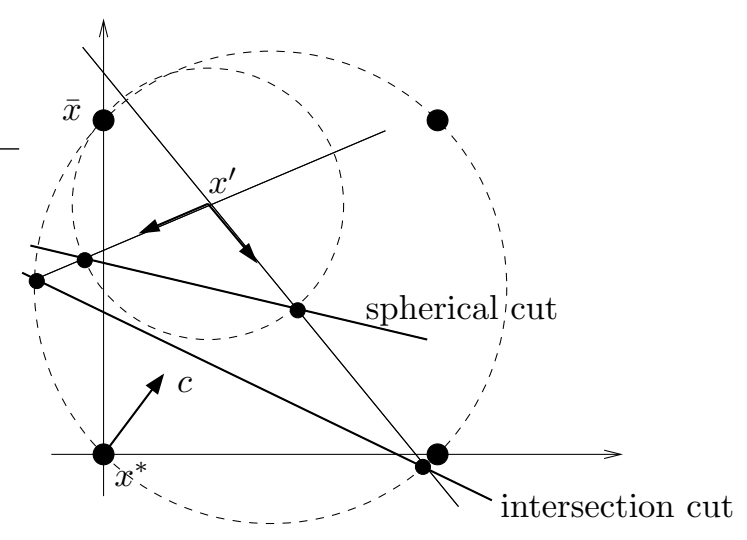

Figure 4: Dominance of intersection over spherical cuts.

If the ball defining the spherical cut is wholly contained within the ball defining the intersection cut, then the intersection cut $\mu x \leq \mu_{0}$ dominates the spherical cut $\pi x \leq \pi_{0}$, as the following result shows. Let $\bar{\sigma}$ be the (filled) ball centered at $y$ with radius $\frac{\sqrt{n}}{2}$, and let $\bar{S}$ be the (filled) ball centered at $x^{\prime}$ with radius $x^{\prime}-\bar{x}$.

\subsection{Proposition}

If $\bar{S} \subseteq \bar{\sigma}$ then the intersection cut for problem (1) at $x^{\prime}$ dominates the corresponding spherical cut on $R$.

Proof. For all $i \leq n$, by construction $\beta_{i}$ belongs to the segment $\left[x^{\prime}, \alpha_{i}\right]$, so the simplex defined by $\left\{x^{\prime}, \beta_{i} \mid i \leq n\right\}$ is wholly contained in the simplex defined by $\left\{x^{\prime}, \alpha_{i} \mid i \leq n\right\}$. Thus, for all $x \in R$ with $\pi x \geq \pi_{0}$ we have $\mu x \geq \mu_{0}$. The result follows.

The proposition above really only applies to very special cases: let $D$ be the set of diagonals of the hypercube cornered in $\lfloor x\rfloor$, i.e. induced by the vertices in $H=\left\{\left\lfloor x^{\prime}\right\rfloor+u \mid u \in\{0,1\}^{n}\right\}$. 


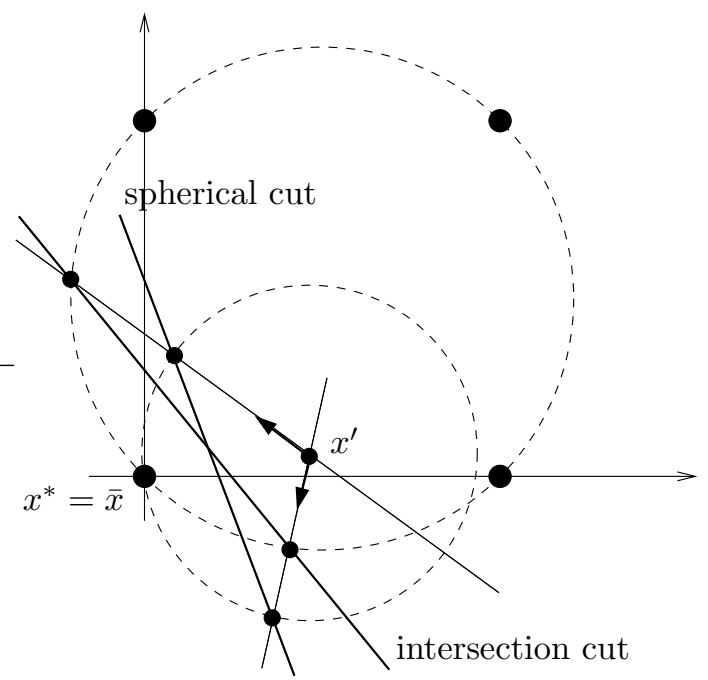

Figure 5: No dominance relation.

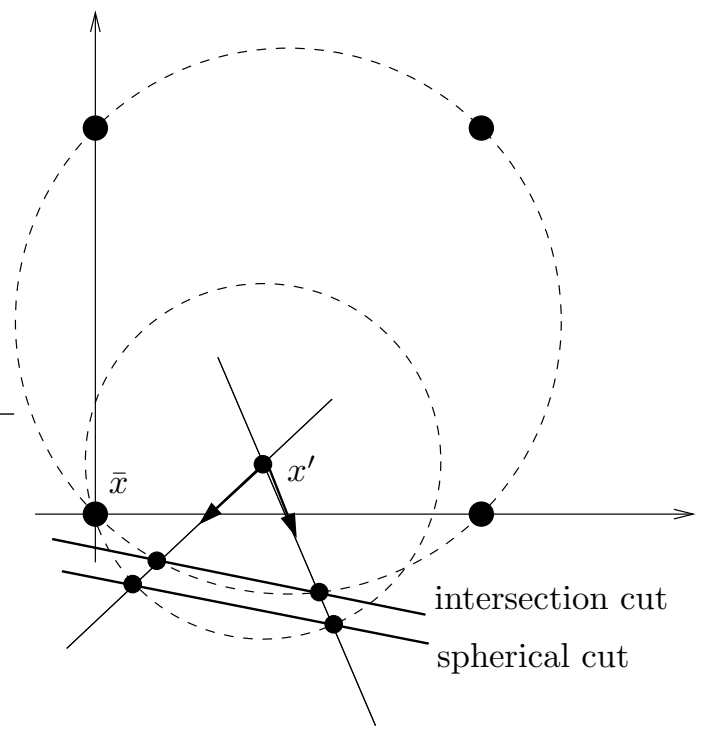

Figure 6: Dominance of spherical over intersection cuts.

\subsection{Lemma}

The locus of points $x^{\prime} \in \bar{\sigma}$ such that $\bar{S} \subseteq \bar{\sigma}$ is $D$.

Proof. Let $x^{\prime} \notin D$. Then $\left\|x^{\prime}-\bar{x}\right\|=\gamma>0$; furthermore, the tangents to $\bar{S}$ and $\bar{\sigma}$ in $\bar{x}$ differ. Hence there must be a neighbourhood of $\bar{x}$ containing a point $z \in \bar{S} \backslash \bar{\sigma}$, proving the result.

The above lemma notwithstanding, there are in practice a lot of cases when intersection cuts dominate spherical cuts in $R$ even though $x^{\prime} \notin D$. 


\subsection{Intersection cuts from outer polars}

In [3], the intersection cut idea was applied to a set generating deeper cuts than those yielded by the sphere $\sigma$ : namely the octahedron $\tau=\left\{x+e \in \mathbb{R}^{n}|| x-e \mid=\frac{n}{2}\right\}$. It is easy to show with examples similar to those above that spherical cuts and intersection cuts from outer polars may be in any type of dominance relation with each other (see Fig. 7).
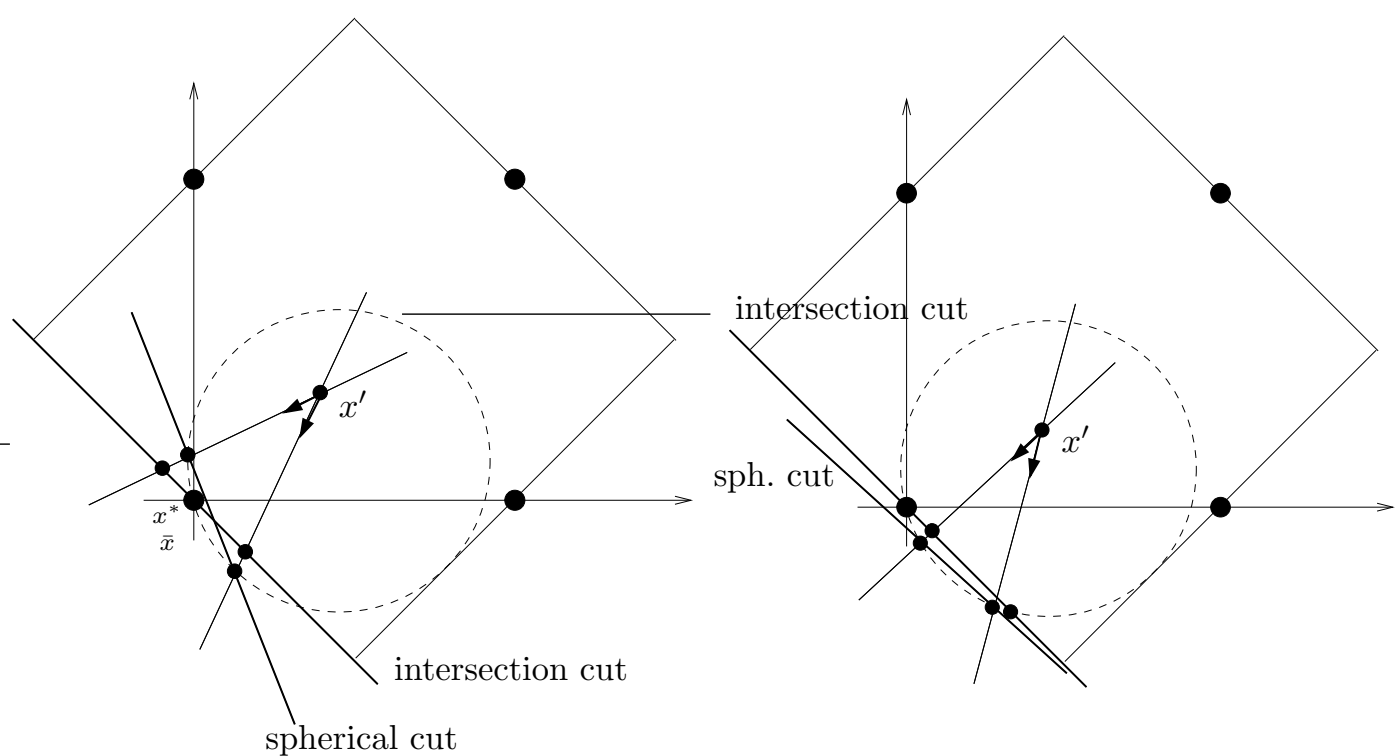

Figure 7: Some dominance relations between spherical cuts and intersection cuts from outer polars. In the picture on the left (no dominance), the negative quadrant is feasible. The picture on the right shows a case where the spherical cut dominates.

\section{Cutting plane algorithm}

A general separation procedure such as spherical cut generation naturally lends itself to be used within a cutting plane algorithm, as follows.

1. Solve the continuous relaxation of (1) to find a solution $x^{\prime}$.

2. If $x^{\prime} \in \mathbb{Z}^{n}$ terminate with solution $x^{\prime}$.

3. Generate a spherical cut $\pi x \leq \pi_{0}$ separating $x^{\prime}$ from $\operatorname{conv}(P)$.

4. Repeat from 1. 
The fundamental question to address is whether such an algorithm converges in a finite number of steps. In this section we show a counterexample showing that the cutting plane algorithm as given above does not, in general, converge to the optimum. Consider the problem:

$$
\left.\begin{array}{rl}
\min -x_{1}-x_{2} & \\
3 x_{1} & \leq 4 \\
3 x_{2} & \leq 4 \\
x_{1}, x_{2} & \geq 0 \\
x_{1}, x_{2} & \in \mathbb{Z} .
\end{array}\right\}
$$

The initial solution of the relaxed problem is $x^{\prime}=\left(\frac{4}{3}, \frac{4}{3}\right)$. The nearest integral point, which also solves the problem, is $x^{*}=(1,1)$. Spherical cuts keep cutting away portions of relaxed feasible region belonging to the circle centered at $x^{\prime}$ with radius $d\left(x^{\prime}, x^{*}\right)$ without ever reaching $x^{*}$, as shown in Fig. 8 .
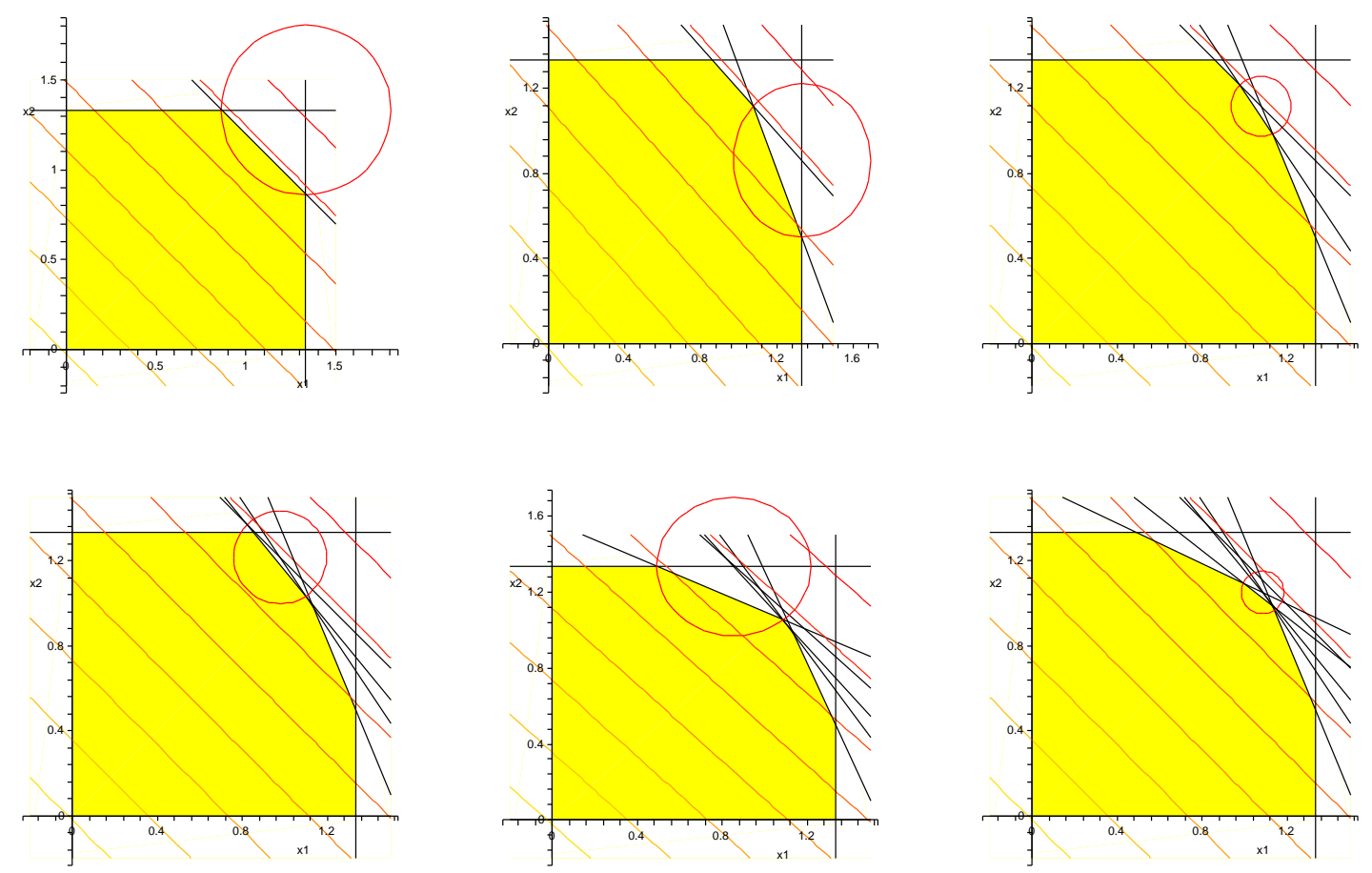

Figure 8: Nonconvergence of the cutting plane algorithm.

In order to show that the cutting plane algorithm does not converge for the example above, we prove that $x^{*}$ does not satisfy any problem constraint or added spherical cut at equality. It follows that $x^{*}$ can never be a vertex of the relaxed polyhedron, which implies the nonconvergence of the algorithm. Since $3 x_{j}^{*}<4$, for $j=1,2$, the original problem constraints are not satisfied at equality by $x^{*}$. It remains to be shown that no spherical cut is ever satisfied at equality by $x^{*}$; this is dealt with in a more general 
setting in Lemma 4.1.

\subsection{Lemma}

Consider problem (1) with optimal (integer) solution $x^{*}$. Assume (a) that $A x^{*}<b$; (b) at each iteration $k$ of the spherical cut based cutting plane algorithm the closest integral point to the the current relaxed solution is always $x^{*} ;(c)$ the first generated spherical cut is not active at $x^{*}$. Then none of the spherical cuts generated during the algorithm is ever active at $x^{*}$.

Proof. We proceed by induction on the cutting plane algorithm iteration index $k$. For $k=1$ the claim is verified by assumption (c). The induction hypothesis is now that for all $j<k$ the claim is verified. Suppose, to get a contradiction, that the $k$-th spherical cut, $\pi x \leq \pi_{0}$, is such that $\pi x^{*}=\pi_{0}$, and let $x^{\prime}$ be the current relaxed solution. By the definition of spherical cut, $x^{*}$ is the intersection of the sphere centered at $x^{\prime}$ of radius $d\left(x^{\prime}, x^{*}\right)$ and a half-line contained in a problem constraint active at $x^{*}$. By (a), no original problem constraint is active at $x^{*}$, so there is an algorithmic iteration $j<k$ such that the spherical cut generated at iteration $j$ is active at $x^{*}$, against the inductive hypothesis.

For problem (5), conditions (a) and (c) of Lemma 4.1 are trivially verified. To verify condition (b), consider Fig. 9, left frame. We have to show that the current relaxed solution $x^{\prime}$ can never be in the sets
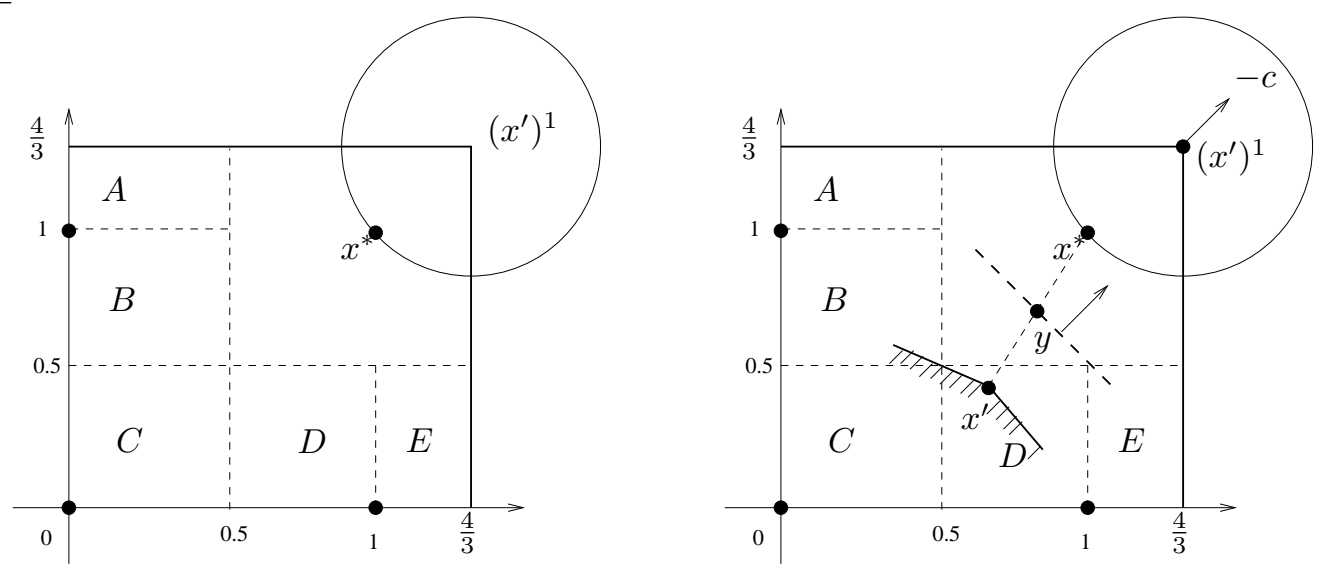

Figure 9: Verification of condition (b) of Lemma 4.1.

$A, B, C, D, E$ (which are closest to integral points different from $x^{*}$ ). Suppose $x^{\prime} \in A \cup B \cup C \cup D \cup E$ (so that $\left.d\left(x^{\prime}, x^{*}\right)>0\right)$. Let $y=\frac{1}{2} x^{\prime}+\frac{1}{2} x^{*}$. Then (by inspection, but it is easy to show it algebraically) $(-c) x^{\prime} \leq(-c) y$ and $(-c) x^{*}>(-c) y$. Hence $(-c) x \leq(-c) y$ separates $R$ (the convex hull of $P$, the feasible region of the integer problem) from $x^{*}$, whence $x^{*}$ is infeasible, against the hypothesis (see Fig. 9, right frame). 


\subsection{Intersection cuts based cutting plane algorithm}

Intersection cuts yield a convergent cutting plane algorithm [2], but only after applying a Gomory-type strengthening of the intersection cut called integerization. If we consider intersection cuts as defined in Sect. 3) with no integerization, a cutting plane algorithm based on them need not converge.

\subsection{Lemma}

Consider problem (1) with optimal (integer) solution $x^{*}$. Assume that $A x^{*}<b$. Then none of the intersection cuts generated during the algorithm is ever active at $x^{*}$.

Proof. The proof, by induction, is very similar to that of Lemma 4.1. By assumption, none of the problem constraints are active at $x^{*}$, which implies that the first generated intersection cut cannot be active at $x^{*}$. For an intersection cut to be active at $x^{*}$ at iteration $k$, one would need at least one relaxed polyhedron edge to be active at $x^{*}$ at iteration $k-1$, but this is impossible by the induction hypothesis, as the polyhedron edges are either original problem constraints or intersection cuts generated at previous iterations.

By Lemma 4.2, problem (5) can also be used to show nonconvergence of an intersection cuts based cutting plane algorithm. So the reason allowing the intersection cut-based cutting plane algorithm to converge is really the integerization procedure applied to the cut.

\subsection{Integerization of spherical cuts}

The integerization procedure described in [2] for intersection cuts applies to

$[\ldots]$ any equation of the simplex tableau that is selected as a pivot row for a dual simplex iteration $[\ldots]$ ([2], p. 27-28)

and hence can readily be applied to spherical cuts as well as intersection cuts. The convergence proof ([2], p. 31-32) specifically depends on intersection cuts only to state that a relaxed fractional solution is cut off by the intersection cut generated at the next iteration. By Thm. 2.2, this also holds for spherical cuts. Hence a spherical cuts based cutting plane algorithm with integerization will converge to the integer solution. 


\subsection{Example}

To conclude the section, we exhibit the behaviour of the spherical cut-based cutting plane algorithm (with no integerization) on the illustrative example below.

$$
\begin{aligned}
\min -2 x_{1}-3 x_{2} & \\
6 x_{1}+4 x_{2} & \leq 5 \\
-x_{1}+2 x_{2} & \leq 1 \\
x_{1}, x_{2} & \in\{0,1\} .
\end{aligned}
$$

With this particular instance, the cutting plane algorithm terminates having found a point $x^{*}$ each component of which is at most $10^{-6}$ away from its closest integral value (see Fig. 10).
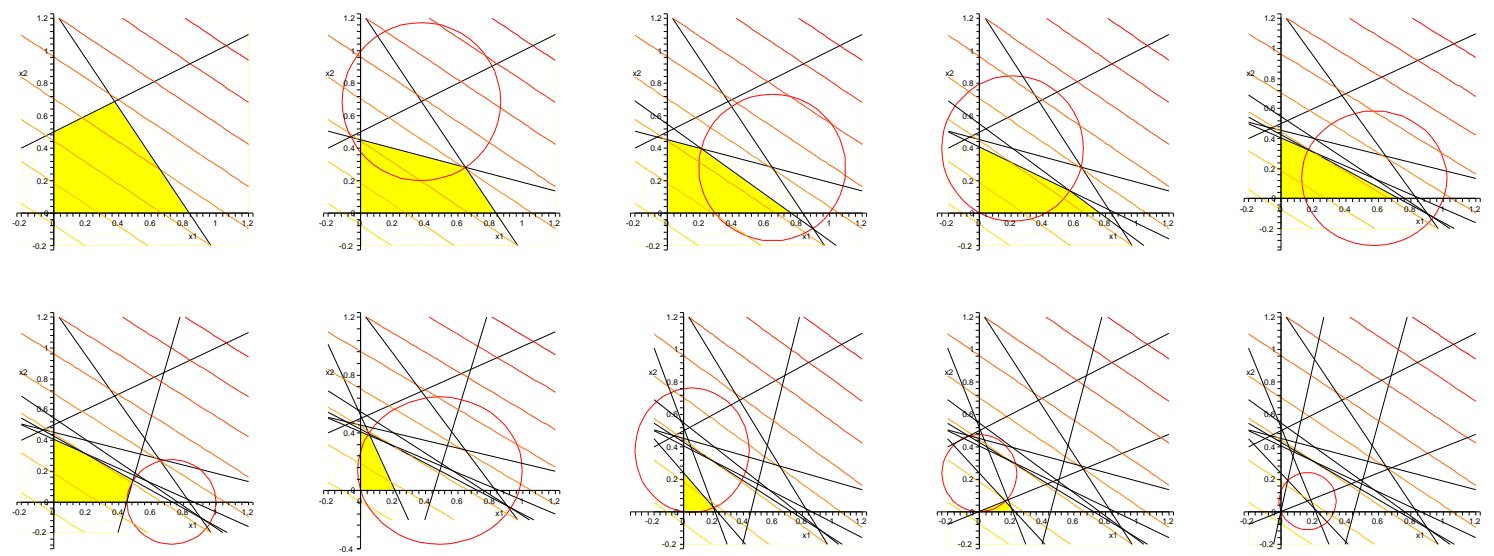

Figure 10: Cutting plane algorithm running to termination on the illustrative example.

\section{Conclusion}

This paper describes a new class of valid cuts, called spherical cuts, which are applicable to general integer programming problems. Spherical cuts are the convex (linear) relaxation of a nonconvex cut consisting of the outside of a sphere centred at the current LP relaxation solution with radius equal to the distance to the nearest integer point. Although the geometric idea underlying spherical cuts is very similar to that of Balas' intersection cuts, no dominance relation can be established between the two classes of cuts. We also show that a cutting plane algorithm based on spherical cuts or intersection cut may fail to converge unless a suitable Gomory-type cut strengthening is put in place. 


\section{Acknowledgements}

The author is grateful to two anonymous referees for helpful comments and suggestions.

\section{References}

[1] E. Althaus, A. Bockmayr, M. Elf, T. Kasper, M. Jünger, and K. Mehlhorn. SCIL - Symbolic constraints in integer linear programming. http://www.mpi-sb.mpg.de/SCIL/scil.ps, 2002.

[2] E. Balas. Intersection cuts - a new type of cutting planes for integer programming. Operations Research, 19(1):19-39, 1971.

[3] E. Balas. Integer programming and convex analysis: intersection cuts from outer polars. Mathematical Programming, 2:330-382, 1972.

[4] E. Balas. Projection, lifting and extended formulation in integer and combinatorial optimization. Annals of Operations Research, 140:125-161, 2005.

[5] E. Balas and R. Jeroslow. Canonical cuts on the unit hypercube. SIAM Journal on Applied Mathematics, 23(1):61-69, 1972.

[6] E.A. Boyd. Fenchel cutting planes for integer programs. Operations Research, 42(1):53-64, 1994.

[7] Y. Chu and Q. Xia. Generating benders cuts for a general class of integer programming problems. In J.-C. Régin and M. Rueher, editors, Proceedings of the 1st International Conference on Integration of AI and OR Techniques in Constraint Programming for Combinatorial Optimization Problems (CPAIOR 2004), volume 3011 of LNCS, pages 127-141, Berlin, 2004. Springer.

[8] R.E. Gomory. Essentials of an algorithm for integer solutions to linear programs. Bulletin of the American Mathematical Society, 64(5):256, 1958.

[9] J.N. Hooker. Constraint satisfaction methods for generating valid cuts. In D.L. Woodruff, editor, Advances in Computational and Stochastic Optimization, Logic Programming, and Heuristic Search, pages 1-30, Dordrecht, 1997. Kluwer.

[10] V. Kaibel and M. Pfetsch. Packing and partitioning orbitopes. Optimization Online, 3(1354), 2006.

[11] A. Letchford and A. Lodi. Strengthening Chvátal-Gomory cuts and Gomory fractional cuts. Operations Research Letters, 30:74-82, 2002.

[12] N. Maculan, E.M. Macambira, and C.C. de Souza. Geometrical cuts for 0-1 integer programming. Technical Report IC-02-006, Instituto de Computação, Universidade Estadual de Campinas, July 2002. 
[13] F. Margot. Pruning by isomorphism in branch-and-cut. Mathematical Programming, 94:71-90, 2002.

[14] F. Margot. Exploiting orbits in symmetric ILP. Mathematical Programming B, 98:3-21, 2003.

[15] G.L. Nemhauser and L.A. Wolsey. Integer and Combinatorial Optimization. Wiley, New York, 1988.

[16] H.D. Sherali and W.P. Adams. A Reformulation-Linearization Technique for Solving Discrete and Continuous Nonconvex Problems. Kluwer Academic Publishers, Dodrecht, 1999. 